\title{
Stellar Associations in the LMC
}

\author{
E. Kontizas, M. Kontizas ${ }^{1}$, D. Gouliermis ${ }^{1}$, A. Dapergolas \\ Astronomical Institute, National Observatory of Athens, P.O. Box \\ 20048, GR-118 10 Athens, Greece
}

\section{R. Korakitis}

National Technical University of Athens, GR-157 80 Zografos, Greece

\author{
D.H. Morgan
}

Royal Observatory of Edinburgh, Scotland EH9 3HJ, UK

\begin{abstract}
The stellar associations are defined as loose unbound concentrations of young stars with a bright $\mathrm{OB}$ stellar component, mainly located at the most recent star forming regions, representing the smallest units in the hierarchy of stellar systems in galaxies. The definition of the associations is discussed and the main properties of their stellar content are summarized. Using plates taken with the $1.2 \mathrm{~m}$ UK Schmidt Telescope a method is developed to detect all stellar associations in the LMC and to find their spatial distribution relative to the LMC's recent star formation history. In $40 \%$ of the LMC region, the number of "single peak" associations has increased considerably $(\sim 3 \times)$. A very interesting result is that the faint limit, where the identified associations are revealed, varies by about $3 \mathrm{mag}$. This indicates not only extinction differences but also the presence of pre-main sequence stars at a wide range of masses.
\end{abstract}

\section{Introduction}

The identification and definition of a stellar association is a difficult task. Any loose group of stars within an area where HII regions and bright $\mathrm{O}$ and B spectral type stars exist, giving evidence of common origin, was accepted as a stellar association (Morgan et al. 1952). In an extended review, Blaauw (1964) described the properties of the associations, which include a variety of objects with a wide range of sizes, often comprising more than one concentration of stars and, in some cases, connected to a star cluster as a bound part of the association. Their dimensions range from those of ordinary galactic clusters to a few hundred parsecs. However "the matter is moreover complicated by the fact that, in some associations, distinct subsystems can be distinguished".

\footnotetext{
${ }^{1}$ Department of Physics, University of Athens, GR-157 83 Zografos, Greece
} 
The need for a more explicit definition of a stellar association was pointed out when such objects were searched in nearby galaxies (Hodge 1986; Efremov et al. 1987; Ivanov 1987; Efremov 1995). It was then realized that different authors use different criteria, which give conflicting sizes of a wide range. It has therefore become clear that various authors do not always refer to the same kind of object and a new definition would be necessary to compare the same kind of stellar system in galaxies (Ivanov 1987; Kontizas et al. 1994; Elmegreen \& Efremov 1996; Bresolin et al. 1998; Elmegreen \& Efremov 1998; Brown 1998).

\section{Current Definition of Stellar Associations}

Stellar associations are single, unbound concentrations of early-type luminous stars, embedded in the most recent star formation regions in a galaxy. The stellar mass density in these systems is accepted to be $\rho \leq 0.1 \mathrm{M}_{\odot} / \mathrm{pc}^{3}$ (Blaauw 1964; Lada \& Lada 1991). Taking into account this definition, we consider the stellar association as a single concentration of stars that can be directly compared to star clusters, either open or globular, providing one extreme end in the various stellar systems in a galaxy. Table 1 illustrates the properties of the main structural parameters of the stellar systems. The open clusters are the intermediate objects and, quite often, they may be confused with stellar associations (Batinelli \& Capuzzo-Doltcetta 1991; Brown 1998).

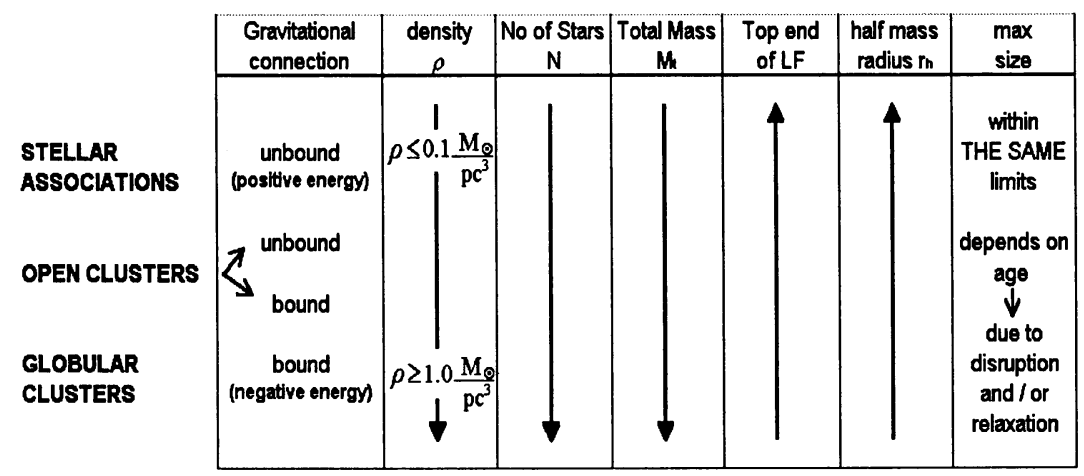

Table 1. Properties of single peak stellar systems

\section{Stellar Associations in the LMC}

The first catalogue of $\mathrm{OB}$ associations in the LMC was published by Lucke \& Hodge (1970, hereafter LH), who listed 122 objects with sizes from 15 to 300 pc and number of stars from a few to 225 , down to a limit of $\mathrm{V}=14.7 \mathrm{mag}$. They pointed out that the most extended associations had star clusters as their members and those would better be called "star clouds". It was also found that $90 \%$ of them coincided with HII regions. Five of those "star clouds" were examined by Kontizas et al. (1994) using star counts and spectral classification of stars, in order to isolate the "single stellar groups". 


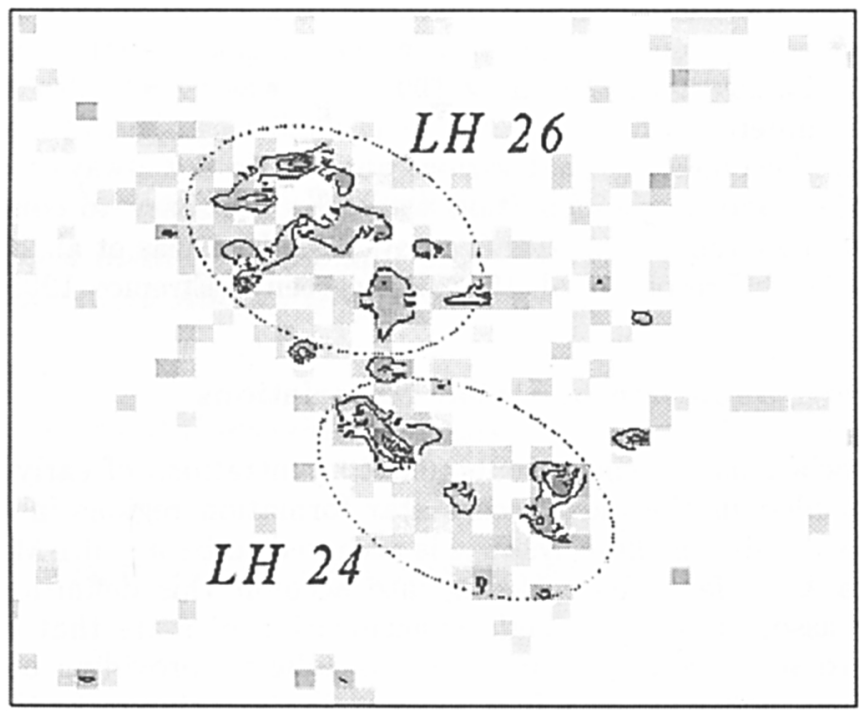

Figure 1. Example of the detection of several associations revealed in the region of the objects LH $24 \& 26$.

\subsection{Stellar Content}

Initial Mass Function. The stellar content of the LMC stellar associations has been investigated by several authors. Being young objects, a major part of their stellar content consists of massive stars, so the age of such systems and the IMF become very important sources of information on the recent star formation and the bright end of the luminosity function (LF).

Garmany (1991) gives an extended review of the previous work, whereas Westerlund (1997) summarizes all recent progress in this direction. The IMF shows a slope $(\gamma)$ from -1.0 to -1.3 (Hill et al. 1994; Massey et al. 1989a,b, 1995), whereas in this meeting Massey (this volume) reports that the slope $\gamma$ is -1.3 for the massive stars in associations of all three galaxies (SMC, LMC and the Milky Way), with no evidence of upper mass cut off.

Stellar Population - Pre-main Sequence Stars (PMS). The associations have been well-known as the primary hosts of very massive stars but, being young in stellar content, they are expected to contain a significant number of PMS which are not visible in the optical wavelengths. Brandl et al. (1996) have observed a region in the core of 30 Doradus (R136), where they detected 110 extremely red sources which are low- or intermediate-mass PMS candidates. The PMS stars were revealed in R136 by Siriani et al. (this volume) from observations with the HST. They found that the red population in the CMD is well tracked by PMS isochrones and it consists of low-mass stars down to $0.8 M_{\odot}$. These stars have ages from 1 to $10 \mathrm{Myr}$, with a flattening from $3 M_{\odot}$ to $1 M_{\odot}$. HST/NICMOS $\mathrm{H}$-band images (Zinnecker, this volume) have shown that the LF rises at least until $\mathrm{H}=20\left(\sim 2 M_{\odot}\right)$ and there are stars much less massive, down to $0.4 M_{\odot}$ 

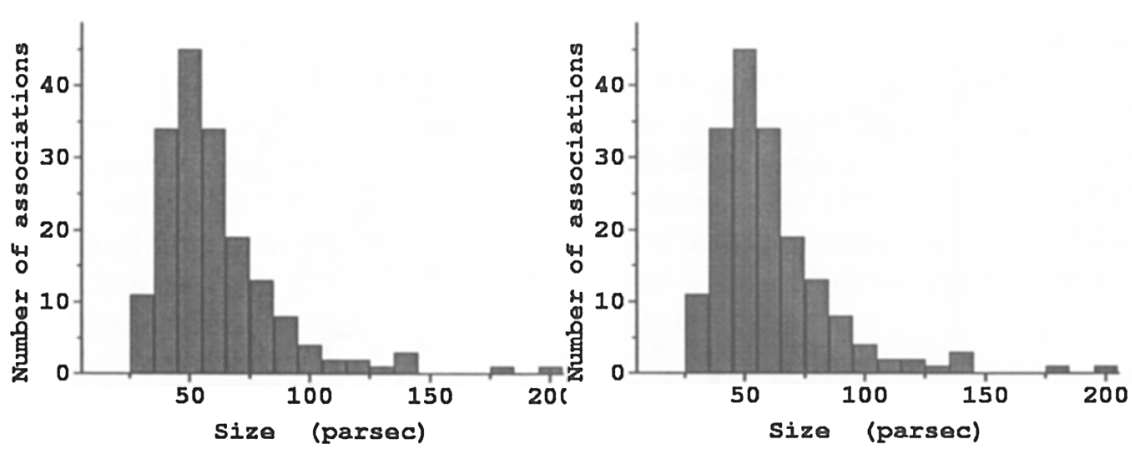

Figure 2. (left) Size distribution and (right) volume density distribution of the detected associations.

in 30 Doradus. There is evidence that PMS stars are identified in this region (Brandner et al., this volume).

Latest observations of the Galactic "associations" and their "subgroups", summarized by Brown (1998), have revealed that the classical membership down to spectral type B8 is corrected and extended to include stars beyond $\mathrm{F}$ type, PMS, T-Tauri and evolved F8, K3 and Wolf-Rayet stars.

Age. The age of the associations is difficult to determine because of the uncertainties in the upper end of the luminosity and its transformation to mass. Depending on the way the mass is determined (photometrically or spectroscopically), for the very massive stars it is particularly sensitive to the observations. In any case, the associations are not found older than a few times $10^{7} \mathrm{yr}$. Often, there is evidence for sequential star formation within a group of nearby associations, such as those of the LH58 which were found to exhibit age differences from 4 - $9 \mathrm{My}$ for the various spatial groups of stars (Hill et al. 1994). The region of LH58 is found to comprise four discrete components (associations), with sizes of about 50,70,100, and $40 \mathrm{pc}$ respectively, showing four different episodes of star formation in space and most probably in time. 30 Doradus, as observed with HST/WFPC2, revealed that "two-stage starbursts" may be characteristic of the massive star formation on this scale (Rubio et al. 1998).

\subsection{Search for "Single" Stellar Associations in the LMC}

We have further developed our method (Kontizas et al. 1994) of detecting all "single peak" stellar OB associations in the LMC, from star counts in very large areas of the LMC at two wavebands: U and HeII (4686 $\AA$ ). Digitized photographic plates, taken with the $1.2 \mathrm{~m}$ UK Schmidt Telescope, have been used and star counts were performed on the image of stars produced by APM and SuperCOSMOS, in order to list all LMC associations according to the current definition.

The magnitudes of the stars detected by the plate scanning procedure were calibrated using as standards the CCD measurements by Will et al. (1996), so that images at various magnitude ranges were produced. Star counts were performed at each magnitude range and statistical tests enabled us to determine the 


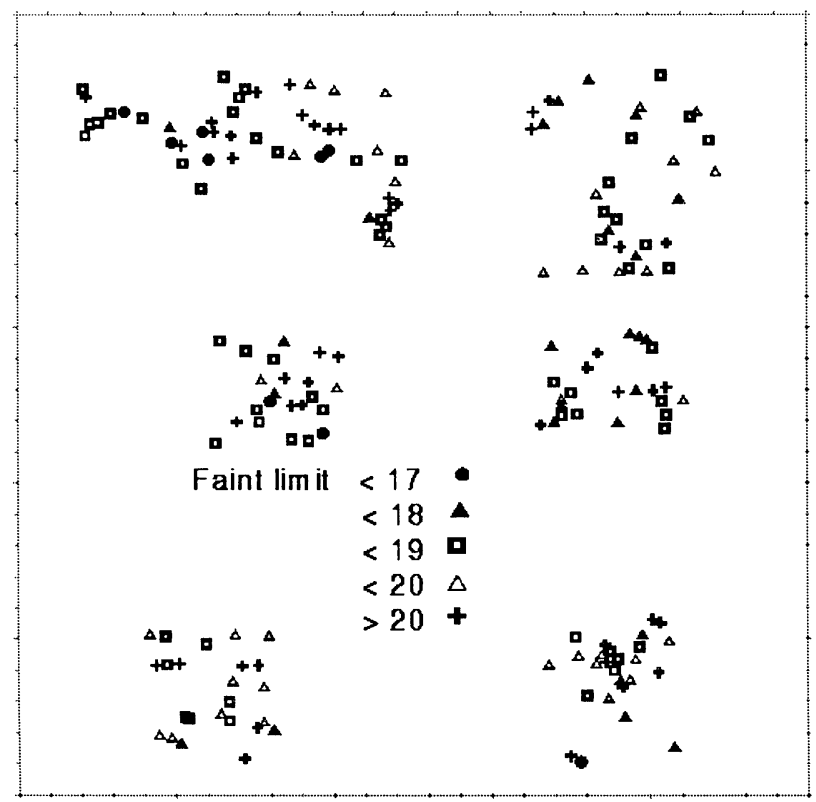

Figure 3. Spatial distribution of the lower detection limit.

background density (number of stars per unit area) and its standard deviation. Using the IRAF \& MIDAS packages, regions with a high star concentration were revealed.

Isodensity contours were produced in each area and the ones corresponding to densities $\geq 3 \sigma$ illustrate the regions where an enhancement of stars is observed. Such isodensity contours in the various magnitude ranges indicate the groupings of the stars at the corresponding magnitudes. This investigation shows the limits at which these stellar groupings are detected (the upper and lower magnitude limit of detection) and whether the bright stars (since the search is for OB associations) show a distinct concentration compared to the background (Fig. 1).

The size adopted for each association is the largest dimension of the outermost isodensity contour $(3 \sigma)$, as described above. Many associations were revealed, bringing their number much higher than in the $\mathrm{LH}$ catalogue. This was expected, since these objects were selected using the new definition of the stellar association. The size distribution of the detected associations is shown in Fig. 2a. This result agrees well with the distribution found by Ivanov (1996) for M31 and M33, and Bresolin et al. (1998) for the nearby galaxies.

The limit of detection of each stellar association at our wavebands showed a wide range of magnitudes, indicating that the low mass stellar component is hidden at different magnitudes, even for the same region on the selected areas (Fig. 3). This effect can be due to extinction but the reddening is never high enough to allow for such a large difference. However, considering that PMS 
stars are expected in these objects, the difference in the limiting magnitude may indicate the existence of PMS stars of different masses in the various associations.

Finally, the detection of the associations in the various magnitude ranges permits the estimation of a mean mass range of the stars found in each object. A total number of stars and, therefore, a total mass within the half mass radius, is calculated. The volume density of each association (Fig. 2b) estimated by this method, even allowing for the existence of low mass stars and/or PMS stars for the adopted IMF (Massey 1999; Zinnecker 1999), gives strong evidence that these objects are in principle loose and unbound.

Acknowledgments. The authors would like to express their sincere thanks to the $1.2 \mathrm{~m}$ U.K Schmidt Telescope Unit for loan of the material and the British Council and the General Secretariat of Research and Technology of Greece for financial support of this project.

\section{References}

Batinelli, P., \& Cappuzzo-Doltcetta, R. 1991, MNRAS, 249, 76.

Blaauw, A. 1964, ARA\&A, 2, 213

Brandl, B., et al. 1996, ApJ, 466, 254.

Bresolin, F. , et al. 1998, AJ 116, 119.

Brown, A. 1998, NATO Advanced Study Institute, May 1998, Crete Greece

Efremov, Y. 1995, AJ, 110, 2757

Efremov, Y., Ivanov, G., \& Nikolov, N. 1987, A\&SS, 135, 119

Elmegreen, B., \& Efremov, Y. 1996, ApJ, 466, 802

Garmany, C. 1991, PASP Conf. Ser., vol. 13

Hodge, P. 1986, IAU Symp. No 116, p. 369

Hill, R.J., Madore, B.F., \& Freedman, W.L. 1994, ApJS, 91, 583

Ivanov, G. 1987, A\&SS 136, 113

Ivanov, G. 1996, A\&A, 305, 1990

Kontizas, M., et al. 1994, A\&AS, 107,77

Lucke, P., \& Hodge, P. 1970, AJ, 75, 171

Massey, P., 1999, this volume

Massey, P., Lang, C.C., Degioia-Eastwood, K., \& Garmany, C.D. 1995, ApJ, 438,188

Massey, P., Parker, J.W., \& Garmany, C.D. 1989b, AJ, 98, 1305

Massey, P., Silkey, M., Garmany, C.D., \& Degioia-Eastwood, K. 1989a, AJ, 97, 107

Morgan, W.W., Sharpless, S., \& Osterbrock, D. 1952, AJ, 57, 3

Rubio, M., Barba, R.H., Walborn, N.R., Probst, R.G., Garcia, J., \& Roth, M.R. 1998, AJ, 116, 1708

Will, J-M., Bomans, D.J., \& de Boer, K.S. 1995, A\&A, 295, 54

Will, J-M., Bomans, D.J., Vallenari, A., Schmidt, J.H.K., \& de Boer, K.S. 1996, A\&A, 315 
Westerlund, B. 1997, "The Magellanic Clouds", Cambridge University Press. Zinnecker, H. 1999, this volume

\section{Discussion}

Philip Massey: Two comments: First, as a user of OB associations I think that a critical test of whether or not an association or group is "physical" is to what extent the member stars are coeval. Although there are a few exceptions, most of the "A1" associations of Lucke and Hodge (1970, AJ, 75, 171) turn out to be highly coeval (Massey et al. 1995, ApJ, 454, 151). You cite the Hill et al. photometric survey of LH58 showing sequential star formation and subclumps of distinctly different ages, but this is simply NOT what we find if we actually take spectra of the bright blue stars and place them on the HRD that way. The cluster was analyzed by Garmany et al. (1994) and rediscussed by Massey et al. (1995, ApJ, 438, 188) along with comparison to the Hill et al. results.

My second comment concerns how reproducable these "subjective" delineations of $\mathrm{OB}$ associations are. I've been blessed with a number of excellent undergraduate REU students over the years, and the current one, Liz Waterhouse, is doing $U B V$ photometry of some of the Lucke-Hodge associations that contain WR stars. On our $15^{\prime} \times 15^{\prime} \mathrm{CCD}$ frames I always have her look at the frame and draw where she sees the OB association. So far her outlines agree very well with those in Lucke's PhD thesis (Lucke 1972, U. Washington). So these A1 associatons are easily distinguished and at some level must be real.

Kontizas: I do not disagree totally since we also found that many of LH associations are single objects, so these must be coeval. To have smaller, single "subgroups" does not necessarily mean they are not coeval too. On the other hand in some areas we expect second generation star formation from one group to the other.

Jan Palouš: If the dissolution time of OB associations derives from (the) spatial density of stars, this number should be different from a critical density for the Milky Way. You give a rather short dissolution time, which may be in contradiction (with) the conclusion of Eva Grebel on the unchanging patterns of star clusters over the past $600 \mathrm{Myr}$.

Kontizas: The clusters as bound systems are not expected to dissolve in time scales of the same order as associations do. The associations are a lot less dense (often $10^{3}$ times less) so their disruption time is much shorter.

Frank Winkler: You mentioned the excellent correlation between Lucke-Hodge associations and HII regions. Have you looked at correlations between your own associations and $\mathrm{H} \alpha$ emission?

Kontizas: No. We have not done this yet. 